\title{
Evaluation of human epidermal growth factor receptor-2 and hormonal receptor expression patterns in breast cancer from fine needle aspiration cytology
}

\section{Mutinda C. Kyama ${ }^{1,3}$, Wairimu Waweru ${ }^{1}$, Mary Mungania ${ }^{2}$, Joshua Nyagol ${ }^{1}$}

${ }^{1}$ Department of Human Pathology, School of Medicine, College of Health Sciences, University of Nairobi, P.O. Box 19676-00202, Nairobi - Kenya

${ }^{2}$ Department of Laboratory Medicine, Kenyatta National Hospital Nairobi - Kenya

${ }^{3}$ Department of Medical Laboratory Sciences, School of Biomedical Sciences, College of Health Sciences, Jomo

Kenyatta University of Agriculture and Technology, P.O. Box 62000-0202, Nairobi City Square - Kenya Corresponding Author

Mutinda C. Kyama, Ph.D

Department of Medical Laboratory Sciences, School of Biomedical Sciences, College of Health Sciences, Jomo Kenyatta University of Agriculture and Technology, P.O. Box 62000-0202, Nairobi City Square - Kenya Tel No: +254 711169526, Email: mkyama@jkuat.ac.ke

\begin{abstract}
Introduction: Breast cancer is the most common cancer among women, accounting for $22 \%$ of all female cancers. In breast cancer, the overexpression of human epidermal growth factor receptor-2 (HER-2/neu) is a prognostic marker indicated for stratification of patients for HER-2/neu targeted therapies, while estrogen receptor (ER) and progesterone receptors $(P R)$ are additional prognostic markers in patients with early stage breast cancer and predictive for response to hormonal therapies. Fine needle aspiration cytology (FNAC), can provide enough cellular sample and evaluation of ER, PR and HER2/neu expression patterns has relevant clinical application because the sample can be collected before surgery and help plan management of breast cancer patients. The aim of the study was to evaluate and classify breast cancer by examining HER-2/neu, ER and PR expression patterns using cellblocks.

Methods: A prospective cross-sectional study was carried out using FNAC samples from 30 patients with breast cancer. The ER, PR and HER2/neu protein overexpression patterns were determined by immunocytochemistry. Breast cancer cases were considered positive for ER and PR when 10\% or more tumor cells were stained. The HER-2/neu overexpression was assessed on a scale of 0 to $3+$ and a score of $3+$ was considered positive.

Results: Estrogen receptor positivity was observed in 50\% of breast cancer cases, while PR positivity in $47 \%$ of the cases and HER2/neu was overexpressed in $13 \%$ of breast cancer cases.

Conclusion: Immunocytochemistry performed on cell block is a feasible method for evaluation of ER, PR and HER2/neu status in breast cancer, especially when the cellblock has adequate tumor cells.

Keywords: Breast cancer, human epidermal growth factor receptor-2, estrogen receptor, progesterone receptor, Immunocytochemistry.
\end{abstract}

\section{Introduction}

Breast cancer comprises of diverse group of neoplasms, which show a broad array of morphological features, genetic changes, and tumor behaviour. ${ }^{[1]}$ These pose a major challenge in patient care as it is difficult to determine the most appropriate therapeutic regimen according to profile of disease characteristics. Given poor prognosis of breast cancer diagnosed at late stages, patient stratification for targeted therapy, ${ }^{[2]}$ remains the most feasible way in improving long survival of patients with breast cancer in Kenya. Breast cancer is the most frequent cancer among women, and has a prevalence of about $22 \%$ of all 
female cancers. ${ }^{[3]}$ Globally over 1 million new cases are reported each year, leading to approximately four hundred thousand breast cancer related death annually. ${ }^{[3]}$ In Kenya, breast cancer has an incidence rate of $13.7 \%$ and is the most prevalent cancer among women, leading to major cause of morbidity and mortality in women. [4]

The over-expression and/or amplification of HER$2 /$ neu in breast cancer is a prognostic molecular marker indicated for patient selection for HER2/neu targeted therapies. ${ }^{[5]}$ The over-expression of HER-2/neu is reported in about 20-30\% of breast cancer and up regulation of HER-2/neu is linked to an aggressive disease process and poor prognosis. ${ }^{[5]}$ A previous study in Kenya reported HER2/neu overexpression in $26 \%$ of breast cancer among women. ${ }^{[6]}$ The steroid hormonal receptors for ER and PR are markers with prognostic value in early stage cancer of the breast and ER predictive for response to hormonal therapies. Estrogen receptors are positive in about $70 \%$ of breast cancer, and are known as estrogen receptor positive breast cancer. ${ }^{[7]}$ Approximately $65 \%$ of breast cancer cases positive for ER will be progesterone receptor (PR)-positive. ${ }^{[7]}$ Studies have reported the overexpression of ER/PR among African breast cancer cases with estimates ranging between $18 \%$ and $72 \% .{ }^{[8,9]}$

Breast cancer, present a clinical, diagnostic and therapeutic challenge. This is because the treatment decision is based on clinicopathological variables that are prognostic in nature, such as size of the tumor, metastasis to the lymph node and histological grade that are not sufficient for implementation of personalized therapy. ${ }^{[5,10]}$ Although advances in breast cancer chemotherapy have achieved a certain rate of success, studies show that breast cancer ER, PR and HER-2/neu positive are difficult to treat and it is now believed that more careful patients stratification using these biomarkers will improve therapeutic outcomes. ${ }^{[5,10]}$ The breast cancer subtypes are classified by assessing the ER, PR and HER2/neu status, which includes luminal A (ER/ PR+, HER-2/neu -), luminal B (ER/ PR+, HER-2/neu +), HER2/neu positive (ER-, PR-, HER-2/neu +) and triple negative breast cancer (TNBC) is negative for ER, PR, and HER2/neu. ${ }^{[1]}$

Tissue biopsies including core needle biopsies have been the samples of choice for evaluating ER, PR and HER-2/neu status. ${ }^{[1]}$ However, the use of fine-needle aspiration cytology samples to evaluate ER, PR and HER-2/neu status using a cell block is recommended, especially in metastatic disease where a biopsy may not be advisable, high cost of core needle biopsy especially in poor resource setting ${ }^{[11-13]}$, or when it is not practical to obtain a surgical excision in patients receiving neo-adjuvant therapy. Furthermore, the benefit of using a cell block is that it is cheaper than core needle biopsy, serial sections can be obtained, the use of same antibodies as those of formalin fixed histological sections ${ }^{[11,12]}$ and is also recommended in case of recurrent disease especially in nodal metastasis.

The use of FNAC for determination of ER, PR and HER-2/neu expression patterns has not been adequately explored in poor resource setting especially in developing countries including Kenya $^{[9,14]}$, furthermore breast cancer stratification into subtypes based on expression patterns of ER, $\mathrm{PR}$ and HER-2/neu using FNAC is lacking. Fine needle aspiration cytology is performed by making several passes within the lump using a needle to obtain the cell sample. ${ }^{[11,13]}$ Fine-needle aspiration cytology is readily available, minimally invasive and has been proven to be safe, fast, and cost effective when compared to core needle biopsies especially for aged or frail patients with co-morbidities and those on follow up for neoadjuvant chemotherapy response. To achieve reliable results FNAC of breast cancer should provide adequate cellular material that is representative of the breast mass. ${ }^{[11-13]}$ Fine-needle aspiration cytology samples may be first used as cellular smear for immediate diagnosis, and residual cells (needle washings) can be obtained by rinsing the needle and preserved for subsequent detection of biomarker by immunocytochemistry. 
Therefore, the proposed research was aimed at assessing the clinical utility of ER, PR and HER$2 /$ neu expression patterns in fine needle aspiration cytology samples from breast cancer patients.

\section{Materials and Methods \\ Patients Study Population}

In this prospective cross-sectional study, a total of 30 fine needle aspiration cytology samples were collected from patients with breast cancer at surgical outpatient clinic of the Kenyatta National Hospital in Nairobi, Kenya between November 2014 and February 2016. All eligible patients recruited in this prospective cross-sectional study were women of 18 years and above who had been diagnosed with breast cancer either through triple assessment (clinical assessment, Imaging techniques (mammography or ultrasonography) and biopsy/fine needle aspiration cytology and had signed a written informed consent before recruitment. Women who had other type of malignancies, or on treatment for breast cancer or those who had undergone mastectomy were excluded. A structured questionnaire was used to interview the participants attending the surgical outpatient (fine needle aspiration) clinic in order to obtain demographic and clinical data as shown in Table 1. The age of the patients ranged from 22 to 100 years, with a mean age of $49.88 \pm 18$ years and median of 48 years (Table 1). The study protocol was approved by ethical and research committee of the University of Nairobi and Kenyatta National Hospital.

\section{Specimen Collection and Cell block preparation}

Fine needle aspiration cytology samples were obtained using a 21 or 23-gauge needle. The material was then placed on a microscopic slide and a second slide was used to spread the material thus obtaining a thin conventional smear, which was immediately fixed in 95\% ethyl-alcohol and stained using both papanicolaou staining and haematoxylin and eosin methods for cytomorphological diagnosis of breast cancer and reported using the national cancer institute $(\mathrm{NCI})$ categorization system. $^{[15]}$

The portion of the remaining aspirate in the needle was used for cell block preparation. Briefly, 10 $20 \mathrm{ml}$ of the aspirate was centrifuged and supernatant discarded. Two drops of pooled plasma were added, followed by gentle shaking. Thereafter, two drops of thromboplastin were added and mixed well to activate clotting factor, followed with two drops of calcium ion. The mixture was left to stand for 5 minutes, followed with transfer of the clot to moistened filter paper. The clot was wrapped well, put in a cassette, and then immediately fixed in $10 \%$ neutral buffered formalin for at least 6 hours. The sample was then processed using the routine tissue processor and embedded to form a cell block.

\section{HER2/neu, ER and PR immunocytochemistry staining}

Immunocytochemical staining for ER, PR and HER2/neu (DAKO, Glastrup, Denmark) was performed on $4 \mu \mathrm{m}$ paraffin sections of cellblock following manufacturer's instructions with minor modification. Briefly, the cellblock sections mounted on slides were deparaffinised by use of xylene followed by alcohol washes. This was followed by heat induced epitope retrieval for 20 minutes. The slides were then blocked by Envision Flex Peroxidase blocking reagent for 5 minutes. They were incubated with primary antibody for 30 minutes and only sections for PR staining were incubated with envision Flex Linker for 15 minutes. The sections were incubated with Envision Flex/HRP for 20 minutes, and incubated with labelled Envision Flex 3,3'-diaminobenzidine (DAB) as a chromogen for 10 minutes, and counterstained with hematoxylin for 5 minutes at room temperature. Between incubations sections were washed with Tris-buffered saline. Coverslipping was performed using the Tissue-Tek SCA coverslipper.

\section{Reporting for immunocytochemistry results}

Allred scoring system was used for ER/PR reporting. ${ }^{[16]}$ Allred score takes into account the percentage of tumour positive cells $(0-5)$ and the intensity of immunocytochemical staining (range 
$0-3)$. The two scores are added to obtain a final score, which may range from 0 to 8 . Tumour cells with a total score of 3-8 are considered positive, whereas those with a score less than 3 are considered negative. Allred scoring stratifies a breast cancer patient's ER/PR status into cancers that are likely to respond to hormone therapy with tamoxifen. ${ }^{[16]}$

The recommendation by the American Society of Clinical Oncology/College of American Pathologists on the HER2/neu -positive status is when (on observing within an area of tumor that amounts to greater than $10 \%$ of contiguous and homogeneous tumor cells) there is evidence of protein overexpression. ${ }^{[17]}$ Score 0 and $1+$ should be interpreted as negative, Score $2+$ must be additionally tested for gene amplification before considering definitive therapy as only about $30 \%$ of these cases show gene amplification which is a prerequisite for definitive therapy. Score $3+$ may be taken as positive as over $90 \%$ of these cases show gene amplification. ${ }^{[17]}$ For cell block adequacy at least 100 tumor cells were counted for assessing the biomarker status. ${ }^{[5,7]}$ Under breast cancer subtypes: Luminal A was defined either ER or PR positive with a negative HER2/neu while, luminal B was either ER positive and or $\mathrm{PR}$ positive with a positive HER2/neu. HER2/neu positive was defined as ER \& PR negative and with HER2/neu positive staining. TNBC was as all ER, PR and HER2/neu receptors staining negative.

\section{Data Analysis and Presentation}

The results are expressed as a mean \pm standard deviation or as median or range. Percentages of the tumor cells stained positive for ER, PR and HER-2/neu immunoreactivity were calculated. Spearman's rank correlation, was performed with 95\% confidence interval. A $P$-value $<0.05$ was considered statistically significant. All data analysis was performed using SPSS, Version 20 (IBM, Armonk, NY, USA).

\section{Results}

All patients were of black descent and majority of breast cancer cases occurred between ages 41-60 years, although breast cancer cases below 40 years had a proportion of $37 \%$ (Table 1). In terms of laterality, $16(53 \%)$ were located on the left breast, and 14 (47\%) on the right breast. Majority of the breast masses were located on the upper outer quadrant (43\%) and the size of breast masses ranged from 2 to $14 \mathrm{~cm}$ (Table 1). The most frequent breast cancer cytomorphologically was ductal carcinoma 27(90\%) (Fig. 1), while axillary nodal positivity was observed in $7(23 \%)$ cases (Table 1).

Estrogen receptor (ER) positivity was observed in $15(50 \%)$ of breast cancer cases, while progesterone receptor (PR) positivity was observed in 14(47\%) of the cases (Fig. 1) and Her-2/neu overexpression was reported in $4(13 \%)$ cases (Fig. 2).

\section{Receptor expression patterns based on} laterality, age, location and size of the breast masses

The ER positivity levels between the left breast and the right breast showed a pearson correlation coefficient, which was significantly higher $(p=0.034)$ in the right breast compared to left breast. The PR positivity levels between the left breast and the right breast showed a pearson correlation coefficient, which was significantly higher $(p=0.011)$ in the right breast than in the left breast. However, no significant correlation was seen with the HER-2/neu expression levels between the left breast and the right breast ( $p$ $=0.683)$. There was no correlation for ER $(p=0.279), \quad \mathrm{PR} \quad(p=0.171) \quad$ and HER-2/neu $(p=0.239)$, overexpression levels between age group as well as ER, PR, and HER-2/neu overexpression levels between different breast mass locations and mass size (Table 2).

Classification of breast cancer cases on the basis of ER, PR and HER-2/neu profile

The Luminal A (ER/PR+, HER-2/neu-) profile subtype consisted of 17 (57\%) cases, while Luminal B (ER/PR+, HER-2/neu+) profile 
subtype consisted of 2 (7\%) on cell blocks. HER2/neu positive breast cancer subtype comprised of $2(7 \%)$ on the cell blocks (Table 3). The triple negative breast cancer characterized by ER-, PR-,
HER-2/neu- profile was observed in 9 (30\%) of the breast cancer cases (Table 3).

Table 1: Clinical characteristics of patients with breast cancer

$\begin{array}{lll}\text { No of Patients } & 30 & \\ \text { Age of patients } & 20-40 & 11(37 \%) \\ & 41-60 & 13(43 \%) \\ & >61 & 6(20 \%) \\ \text { Location of primary breast mass } & \text { Left breast } & 16(53 \%) \\ & \text { Right breast } & 14(47 \%) \\ \text { Location of the largest breast } & \text { Upper Outer quadrant (UOQ) 13(43\%) } \\ & \text { Upper Inner quadrant (UIQ) 6(20\%) } \\ & \text { Lower outer quadrant (LOQ) 5(17\%) } \\ \text { Lize of the largest breast mass } & \text { Lower inner quadrant (LIQ) } 3(10 \%) \\ & \text { Periareolar } & 3(10 \%) \\ & <2 \mathrm{~cm} & 2(7 \%) \\ & 3-6 \mathrm{~cm} & 16(53 \%) \\ \text { Cytodiagnosis of conventional smear } & 7-10 \mathrm{~cm} & 6(20 \%) \\ & 11-14 \mathrm{~cm} & 6(20 \%) \\ & \text { Ductal carcinoma } & 27(90 \%) \\ \text { Nodal status at diagnosis } & \text { Mucinous carcinoma } & 2(7 \%) \\ & \text { Suspicious Lobular carcinoma } & 1(3 \%) \\ & & 7(23 \%)\end{array}$

Table 2: Receptor status based on laterality, location and size of the breast masses ( $\mathrm{n}=30$ )

$\begin{array}{llll}\text { n } & \text { ER+ } & \text { PR+ } & \text { HER2+ }\end{array}$

Age of patients:
\[ \begin{array}{r}20-40 \\ 41-60 \\ >61\end{array} \]

Laterality of the breast mass Left

Right

11
13
6

13

16

14

Location of the breast mass

Upper outer quandrant

Upper inner quandrant

Lower outer quandrant

Lower inner quandrant

Periareolar

13
6
5
3
3

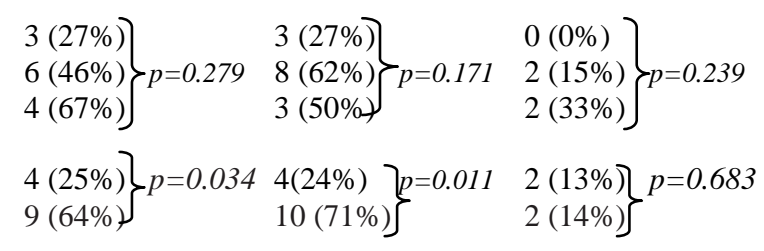

Size of the breast mass

$\left.\left.\left.\begin{array}{lll}>2 \mathrm{~cm} & 2 & 1(50 \%) \\ 3-6 \mathrm{~cm} & 16 & 6(38 \%) \\ 7-10 \mathrm{~cm} & 6 & 4(67 \%) \\ 11-14 \mathrm{~cm} & 6 & 2(33 \%)\end{array}\right\} \begin{array}{ll}0(0 \%) \\ p=0.820 & 3(50 \%) \\ 3(50 \%) & 3(50 \%)\end{array}\right\} \begin{array}{l}1(50 \%) \\ 1(6 \%) \\ p=0.908 \\ 1(17 \%) \\ 1(17 \%)\end{array}\right\} p=0.736$

ER, estrogen receptor, PR, progesterone receptor, HER-2/neu, human epidermal growth factor receptor 2

Interparametric correlation with ER,PR and HER-2/neu (*pearson correlation coefficient) 


\section{JMSCR Vol||06||Issue||07||Page 675-683||July}

Table 3: Classification of breast cancer cases on the basis of their ER, PR and HER-2/neu profile on cell block $(\mathrm{n}=30)$

\begin{tabular}{ll}
\hline ER+, PR+, HER-2/neu- & $17(57 \%)$ \\
ER+, PR+, HER-2/neu+ & $2(7 \%)$ \\
ER-, PR-, HER-2/neu+ & $2(7 \%)$ \\
ER-, PR-, HER-2/neu- & $9(30 \%)$
\end{tabular}

$\overline{\mathrm{ER}}$, estrogen receptor, PR, progesterone receptor, HER-2/neu, human epidermal growth factor receptor 2
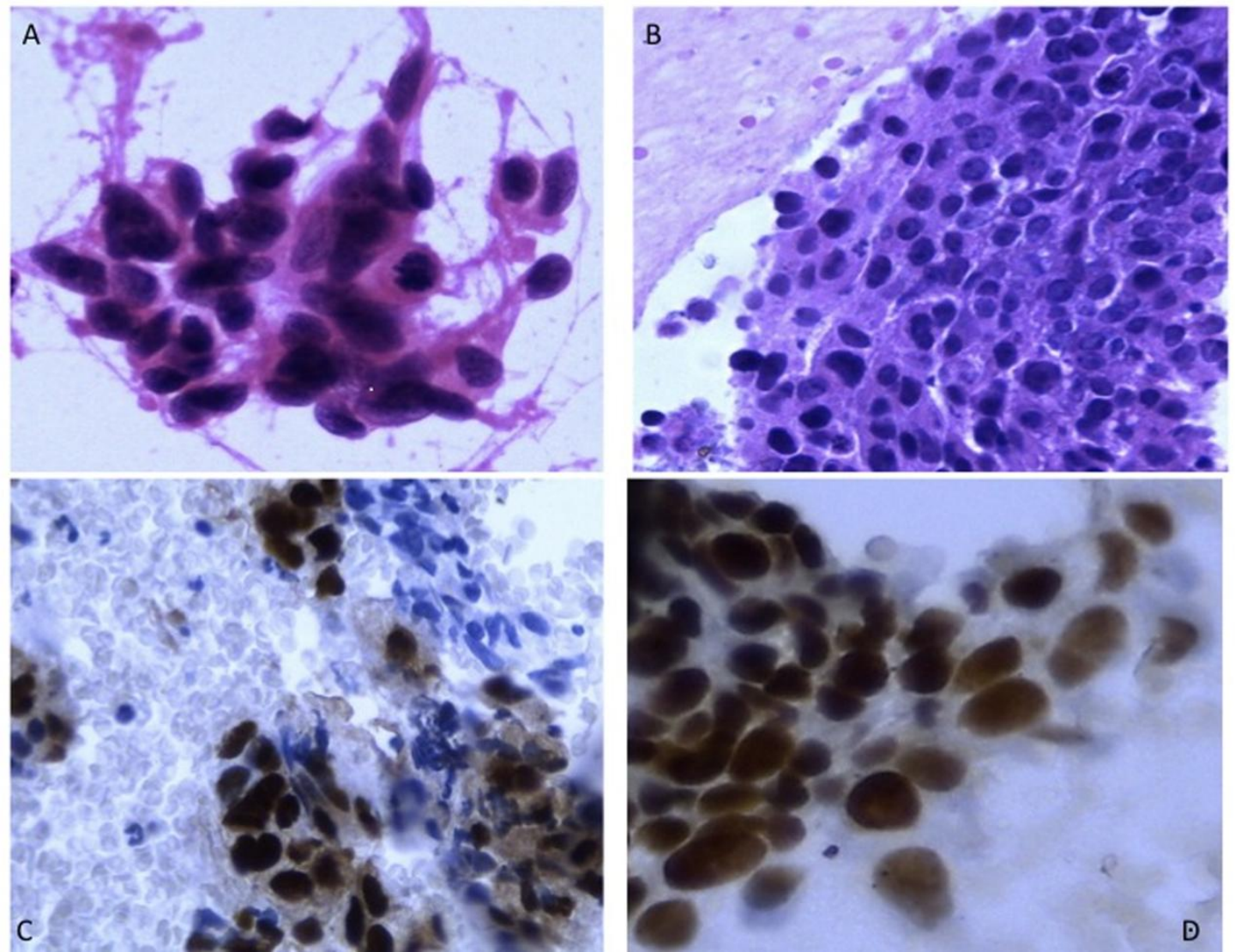

Fig. 1: A. Fine needle aspiration cytology (conventional smear) with discohesive clusters of malignant ductal cells, with marked nuclear pleormophism, hyperchromasia and coarse chromatin (haematoxylin and eosin); B. cell block section with clusters of malignant ductal cells, with marked nuclear pleormophism (haematoxylin and eosin). C. Progesterone receptor immunostaining on cellblock section. A strong positive reaction for PR is seen on the nuclei of cancer cells (proportion $=3$, intensity $=3$ ) $(x 400)$. D Estrogen receptor immunostaining on a cell block section. A strong positive reaction for ER is seen on the nuclei of cancer cells (proportion $=5$, intensity $=3)(x 400)$. 


\section{JMSCR Vol||06||Issue||07||Page 675-683||July}
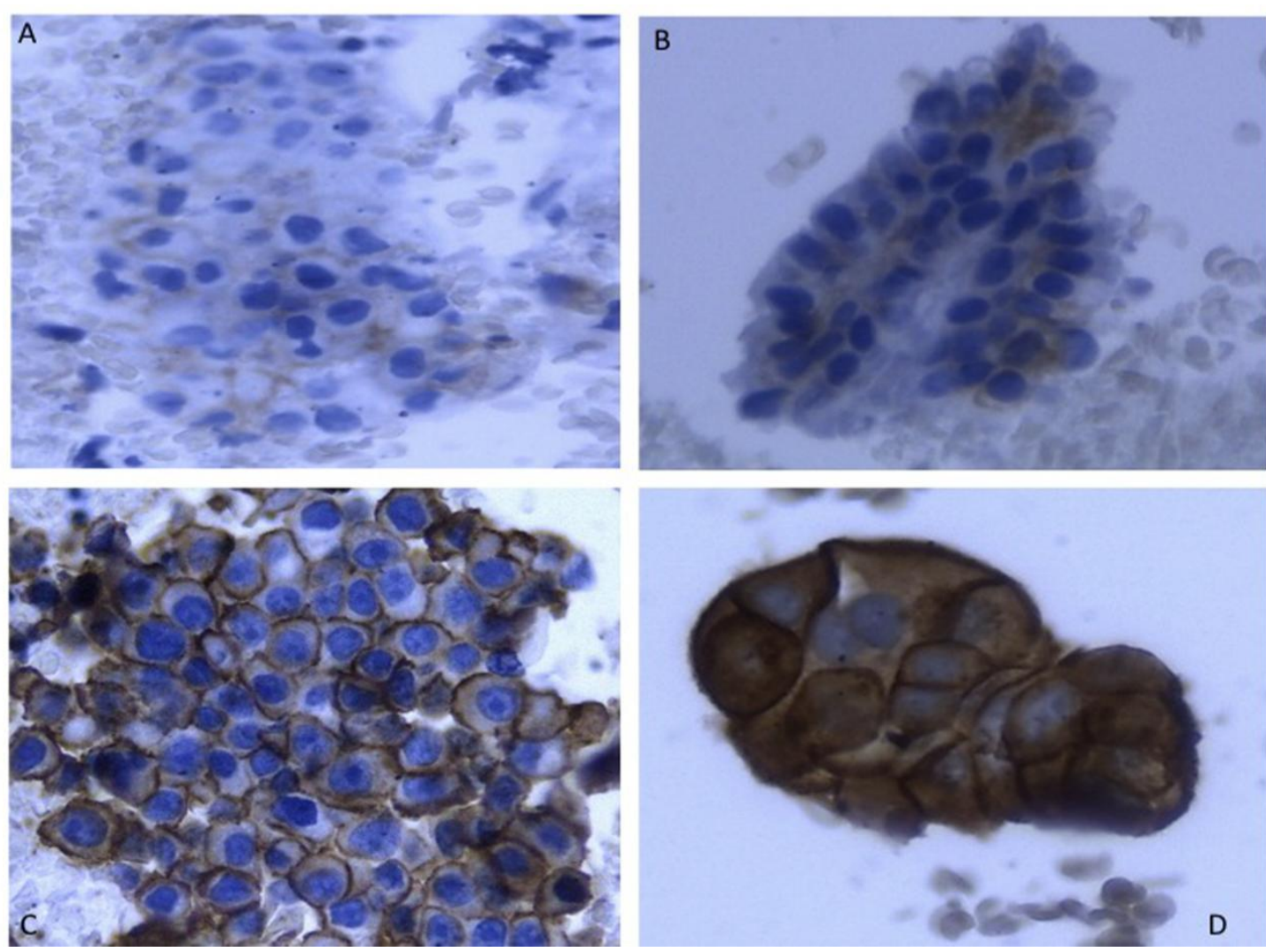

Fig.2: HER2/neu performed on cell blocks. A,B. Her2-negative (1+), partial weakly noncircumferential staining, X400. C,D. HER2/neu immunostaining on a cell block in a HER2/neu over-expressed breast cancer case. Strong complete membrane and intense staining on the tumor cells of HER2/neu (score $3+$ ) is observed (x400).

\section{Discussion}

This study has demonstrated that it is possible to determine the expression patterns of ER, PR and HER2/neu in breast cancer using cell block prepared from FNAC for targeted therapy. This study reported ER positivity in $50 \%$ of breast cancer cases, while PR positivity was reported in $47 \%$ of cases. These expression patterns are within the range of earlier studies, which reported overexpression of ER/PR among African breast cancer cases with estimates ranging between $18 \%$ and $72 \% \cdot{ }^{[6,8,9,14]}$ Human epidermal growth factor receptor-2 (HER-2/neu) was positive in $13 \%$ of breast cancer cases using cell block. The frequency of HER2/neu overexpression varies in Kenyan studies, Bird et al reported HER2/neu overexpression in $26 \%$ of breast cancer cases among women in Kenya, ${ }^{[6]}$ Nyagol et al. $20.26 \%,{ }^{[8]}$ while a recent study by Sayed et al showed $17 \%$ of HER2/neu overexpression by immunohistochemistry. ${ }^{[9]}$
The current study reported a $57 \%$ proportion of the luminal A (ER+, PR+, HER2/neu-) profile of breast cancer using a cell block. A previous study showed a lower proportion (38\%) of luminal A in a cell block. ${ }^{[18]}$ While the luminal $\mathrm{B}(\mathrm{ER}+, \mathrm{PR}+$, HER2/neu+) profile of breast cancer subtype comprised of $7 \%$ on cell blocks which was slightly higher than earlier study which reported $6 \%$ proportion of luminal B using a cell block. ${ }^{[18]}$ The proportion (7\%) of HER2/neu positive breast cancer subtype in our study was lower than previous work which reported a proportion of $24 \%$ using a cell block. ${ }^{[18]}$ The triple negative breast cancer (TNBC) characterized by lack of expression of ER, PR and HER2/neu was reported in $30 \%$ of the cases, which is similar to Kurmar et al., study which reported $32 \%$ proportion of TNBC in cell block. ${ }^{[18]}$ Previous study from Mali reported high TNBC of $46 \%$ of breast carcinomas using biopsies. ${ }^{[19]}$ 
The data of this study has demonstrated that it is possible to determine the expression patterns of ER, PR and HER-2/neu in breast cancer using cell block prepared from FNAC for targeted therapy. This study is important because previous studies reported simultaneous evaluation of ER and PR status by ICC on cellular material obtained by FNAC, which demonstrated high correlation with results from paraffin sections. ${ }^{[11]}$ Furthermore, the use of immunocytochemistry to assess ER status on paired FNAC and paraffin embedded tissues, showed a reliable correlation, with a range of $61 \%$ to $92 \%$ rate of concordance. ${ }^{[20]}$

Therefore, this study has demonstrated that the use of FNAC samples to assess HER-2/neu, ER or PR status using a cell block is useful, especially where disease has metastasized and a biopsy may not be advisable. ${ }^{11-13}$ Also use of cell blocks in developing countries with poor resources is feasible since it is cheaper than core needle biopsy, serial sections can be obtained and use of same antibodies as those of formalin fixed histological sections. ${ }^{11-13} \mathrm{We}$ were unable to compare with paired histological biopsies due to unavailability of most samples either from core needle biopsies or excisional biopsies. Although our preliminary data are interesting, due to lack of comparison with biopsy we were unable to control for false negative results which have been reported in previous studies. ${ }^{[18]}$ Also due to small sample size used in this study confirmation of these data in a larger and independent patient population is recommended. In conclusion, immunocytochemistry performed on cellblock is a feasible method for evaluation of ER, PR and HER-2/neu status of breast cancer, especially when cellblock has adequate tumour cells.

\section{Acknowledgements}

We acknowledge the staff and nurses of the surgical outpatient clinic and the histology and cytology laboratory of the Kenyatta National Hospital in Nairobi Kenya for helping in collection of samples. We thank technical staff of the histopathology laboratory in the Department of Human Pathology of the University of Nairobi for helping in the immunocytochemistry experiments. This work was supported by AFRICA-ai-JAPAN innovation research grant.

\section{References}

1. Simpson PT, Reis-Filho, JS, Gale T et al. Molecular evolution of breast cancer. $J$ Pathol 2005; 205: 248-254.

2. Goldhirsch A, Winer EP, Coates AS et al. Personalizing the treatment of women with early breast cancer: highlights of the $\mathrm{St}$ Gallen International Expert Consensus on the Primary Therapy of Early Breast Cancer 2013. Ann Oncol 2013; 24: 2206-2223.

3. DeSantis C, Ma J, Bryan L, et al. Breast cancer statistics, 2013. CA Cancer J Clin. 2014; 64 :52-62.

4. WHO Cervical cancer summary report update. September, 15: 2010.

5. Durgapal P, Mathur SR, Kalamuddin M, et al. Assessment of Her-2/neu status using immunocytochemistry and fluorescence in situ hybridization on fine-needle aspiration cytology smears: experience from a tertiary care centre in India. Diagn Cytopathol. 2014;42:726-731.

6. Bird PA, Hill AG, Houssami N. Poor hormone receptor expression in East African breast cancer: evidence of a biologically different disease? Ann Surg Oncol 2008;15:1983-8.

7. Geethamala K, Murthy VS, Vani BR, et al. Comparison of Immunocytochemistry and Immunohistochemistry on Breast Carcinoma: A Boon or a Bane? J Lab Physicians. 2017 Jan-Mar;9(1):5-10.

8. Nyagol J, Nyong'o A, Byakika B et al. Routine assessment of hormonal receptor and her-2/neu status underscores the need for more therapeutic targets in Kenyan women with breast cancer. Anal Quant Cytol Histol 2006; 28:97-103.

9. Sayed S, Moloo Z, Wasike R, et al. Is breast cancer from Sub Saharan Africa truly 
receptor poor? Prevalence of ER/PR/HER2 in breast cancer from Kenya. Breast 2014; 23:591-6.

10. Strasser-Weippl K and Goss PE. Advances in adjuvant hormonal therapy for postmenopausal women. J Clin Oncol 2005; 23: 1751-1759.

11. Bueno Angela SP, Viero RM, Soares CT, et al., Fine needle aspirate cell blocks are reliable for detection of hormone receptors and HER2 by immunohistochemistry in breast carcinoma. Cytopathology. 2013;24:26-32.

12. Srivastava P, Kumar B, Joshi U, et al. To Evaluate the Applicability of Parameters of Cytological Grading Systems on Aspirates of Breast Carcinoma. J Cytol. 2018 JanMar;35(1):15-21

13. Mišković J, Zorić A, Radić Mišković H, et al. Diagnostic Value of Fine Needle Aspiration Cytology for Breast Tumors Acta Clin Croat. 2016 Dec;55(4):625-628

14. Nyagol J, Kisato V, Ochuk W et al. Assessment of hormonal receptors and Her2/neu status in breast cancer using cell block: a case study. J. Afr Cancer 2013; 5: 180-184.

15. Gorman BK, Kosarac O, Chakraborty S, et al. Comparison of breast carcinoma prognostic/predictive biomarkers on cell blocks obtained by various methods: Cellient, formalin and thrombin. Acta Cytol. 2012;56:289-96.

16. Allred DC, Harvey JM, Berardo $\mathrm{M}$ et al. Prognostic and predictive factors in breast cancer by immunohistochemical analysis. Mod Pathol 1998;11: 155-68.

17. Wolff AC, Hammond ME, Hicks DG et al. Recommendations for Human Epidermal Growth Factor Receptor 2 Testing in Breast Cancer. Arch Pathol Lab Med. 2014; 138: 241-256.
18. Kumar SK, Gupta N, Rajwanshi A et al. Immunochemistry for oestrogen receptor, progesterone receptor and HER2 on cell blocks in primary breast carcinoma. Cytopathol 2012; 23:181-186.

19. Ly M, Antoine M, Dembélé AK, et al. High incidence of triple-negative tumors in subsaharan Africa: a prospective study of breast cancer characteristics and risk factors in Malian women seen in a Bamako university hospital. Oncology 2012; 83:257-63.

20. Schmitt FC, Bento MJ, Amendoeira I. Estimation of estrogen receptor content in fine-needle aspirates from breast cancer using the monoclonal antibody 1D5 and microwave oven processing: correlation with paraffin embedded and frozen sections determinations. Diagn Cytopathol 1995; 13:347-51. 